\title{
Connecting Healthcare Providers With Patients Through Mobile Technology: Formula for Shared Decision Making and Improved Patient Outcomes
}

Elaine Rudell ${ }^{1}$, CHCP; Patty Peterson ${ }^{1 *}$, CHCP; Sandeep Pulim ${ }^{1 *}$, MD; Andrea L Griffin ${ }^{2 *}$; James D Bowen ${ }^{3 *}$, MD

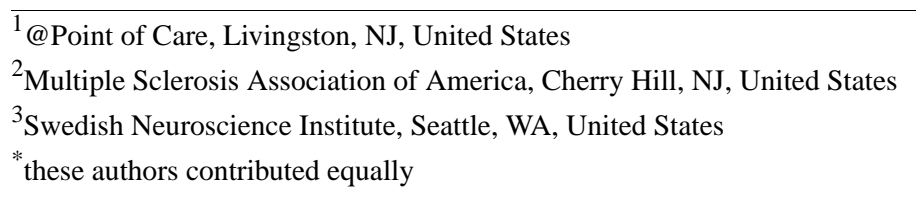

Corresponding Author:

Elaine Rudell, CHCP

@ Point of Care

290 West Mt. Pleasant Avenue, Suite 2350

Livingston, NJ, 07039

United States

Phone: 19738908988 ext 113

Fax: 19739925830

Email: e.rudell@projectsinknowledge.com

\begin{abstract}
Background: Integration of mobile devices/health-related apps into medical practice is transforming healthcare. A 2015 Healthcare Information and Management Systems Society (HIMSS) technology survey of 238 respondents revealed $~ 90 \%$ of healthcare providers utilize mobile devices to engage patients in their healthcare. A new app for clinicians, Multiple Sclerosis @ Point of Care powered by IBM Watson, paired with the Multiple Sclerosis Association of America (MSAA) patient app, My MS Manager, is a practice-based tool designed to provide shared decision making between the clinician and patient, syncing patient data via a Health Insurance Portability and Accountability Act (HIPAA)-compliant mobile cloud platform. The Multiple Sclerosis@Point of Care IBM Watson cognitive learning tool answers questions clinicians pose at point of care to improve patient outcomes.
\end{abstract}

Objective: This analysis evaluates how clinicians use Multiple Sclerosis @ Point of Care, utilize our trained IBM Watson corpus, and benefit from the app. Additionally, the analysis assesses how patients use and benefit from the patient app and how both apps are used for shared decision making to improve patient care.

Methods: To assess how clinicians utilize/value the Multiple Sclerosis @ Point of Care and patient app as well as how patients connect with their clinicians utilizing the patient app, data collected from participating clinicians $(\sim 11,000+)$ caring for multiple sclerosis (MS) patients and their participating patients were analyzed. The My MS Manager patient app provides a tool for patients to record their daily data regarding MS management and share this data with their clinician. Additionally, the clinician can access evidence-based answers at the time it is needed from the Multiple Sclerosis @ Point of Care clinician app. Together, these facilitate shared decision-making at the point of care. Data included demographic information, clinician and patient monthly access frequency, clinicians' questions posed to Multiple Sclerosis @ Point of Care's Ask Watson cognitive tool, clinicians' self-reported impact of content on their patients' health outcomes, number of registered patient app users, average active patient users/month, patient access frequency, patient record entries (including fatigue scale records), and patient perceived benefits.

Results: Overall findings include that (1) clinicians and their patients use the Multiple Sclerosis @ Point of Care clinician app and MSAA patient app, respectively, enabling clinicians to sync with their patients' data to improve outcomes; (2) many clinicians agree the clinician app, Multiple Sclerosis @ Point of Care, and the synched patient data from the My MS Manager app provide timely, relevant information that positively impacts their patients' health outcomes; (3) increasing numbers of patients are using the patient app to enter their data, track their MS management, and share this data with their clinicians; and (4) the fatigue scale entries continue to increase and represent valuable information for their treating clinicians. Frequency of use reported by patients responding to this question show $77 \%$ access the patient app daily or weekly. Better fatigue management resulting from the use of the patient app was reported by $75 \%$ of patients responding to the survey. 
Conclusions: Clinicians engaged in learning utilizing Multiple Sclerosis @ Point of Care currently number 10,627 unique users who spend an average of 8 minutes per visit. Around 86\% of My MS Manager patient app users revealed the app has motivated them to discuss their MS management with their clinician. Management of MS is evolving rapidly and the findings of this analysis show Multiple Sclerosis @ Point of Care and the My MS Manager patient app facilitate the interface of clinicians and MS patients for shared decision making that support strategies for practice change and improved patient outcomes through point of care accessibility.

(iproc 2016;2(1):e3) doi: 10.2196/iproc.6224

\section{KEYWORDS}

multiple sclerosis; shared decision-making; mobile apps; mHealth

This poster was presented at the Connected Health Symposium is displayed as an image in Figure 1 and as a PDF in Multimedia 2016, October 20-21, Boston, MA, United States. The poster Appendix 1.

Figure 1. Poster.

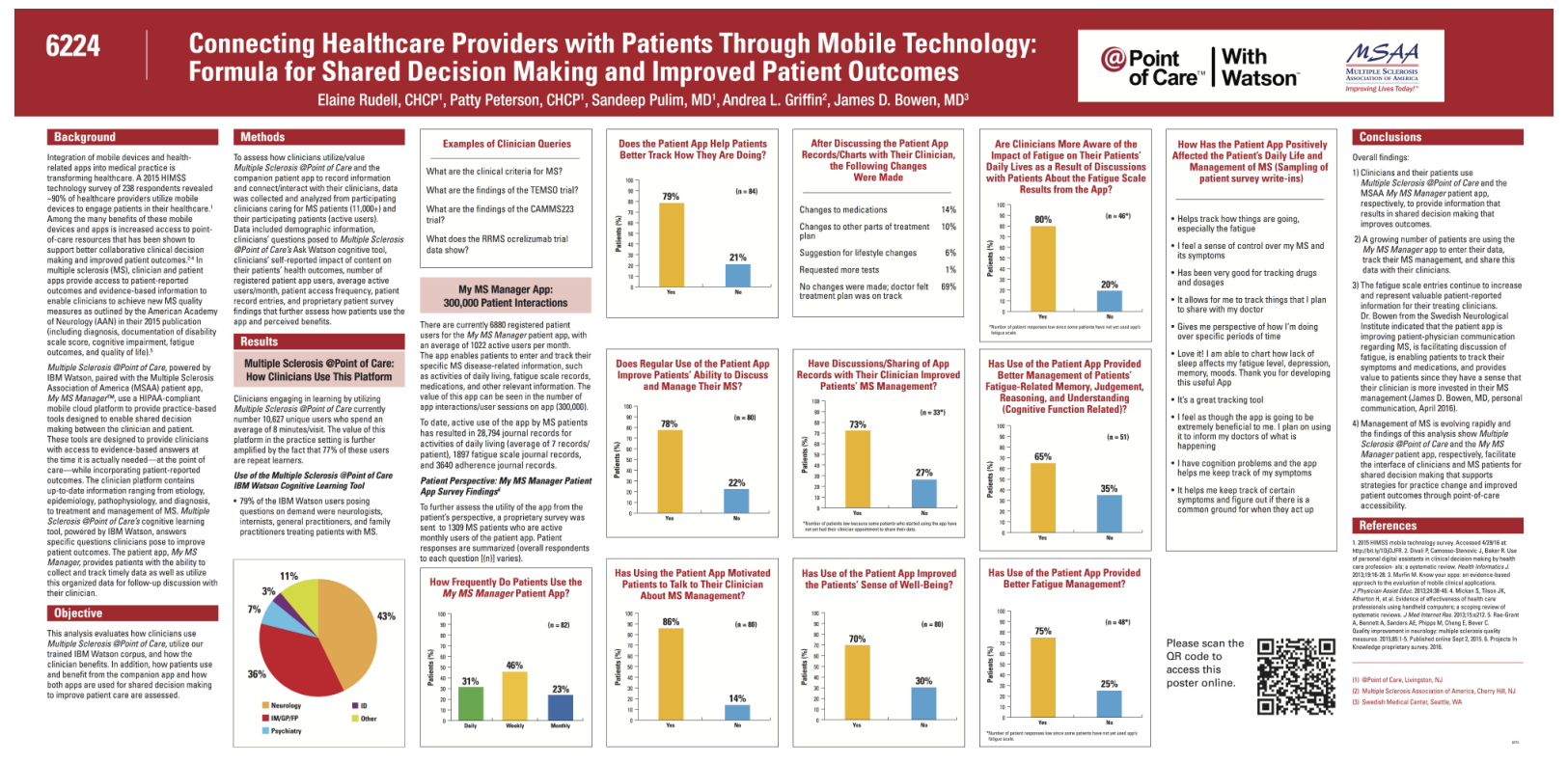

\section{Multimedia Appendix 1}

Poster.

[PDF File (Adobe PDF File), 767KB-Multimedia Appendix 1]

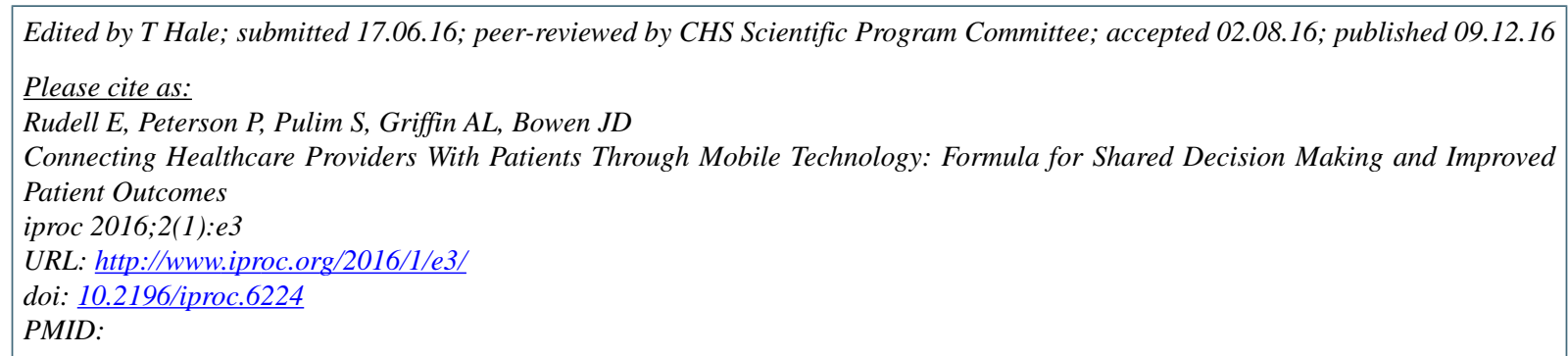

CElaine Rudell, Patty Peterson, Sandeep Pulim, Andrea L Griffin, James D Bowen. Originally published in Iproceedings (http://www.iproc.org), 09.12.2016. This is an open-access article distributed under the terms of the Creative Commons Attribution License (http://creativecommons.org/licenses/by/2.0/), which permits unrestricted use, distribution, and reproduction in any 
medium, provided the original work, first published in Iproceedings, is properly cited. The complete bibliographic information, a link to the original publication on http://www.iproc.org/, as well as this copyright and license information must be included. 